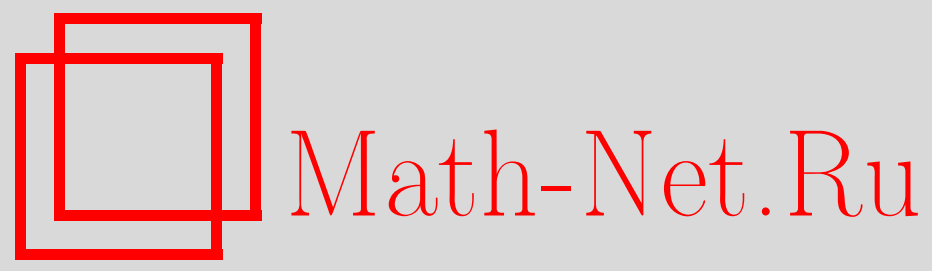

О. В. Камозина, Алгебраические решетки кратно $\Omega$ расслоенных классов Фиттинга, Дискрет. матем., 2006, том 18, выпуск 2, 139-145

DOI: https://doi.org/10.4213/dm53

Использование Общероссийского математического портала Math-Net.Ru подразумевает, что вы прочитали и согласны с пользовательским соглашением http: //www .mathnet.ru/rus/agreement

Параметры загрузки:

IP : 54.198 .64 .247

26 апреля 2023 г., $11: 45: 32$

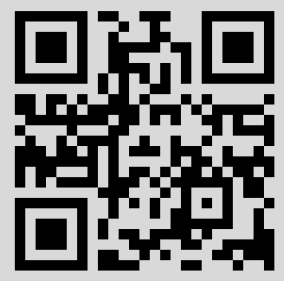




\title{
Аискретная математика
}

том 18 выпуск $2 * 2006$

Удк 512.542

\section{Алгебраические решетки кратно $\Omega$-расслоенных классов Фиттинга}

\author{
() 2006 г. О. В. Камозина
}

\begin{abstract}
В статье изучаются решетка всех классов Фиттинга, решетка всех $n$-кратно $\Omega$-расслоенных классов Фиттинга с направлением $\varphi, \psi_{0} \leqslant \varphi$, и решетка всех тотально канонических классов Фиттинга. Показано, что данные решетки являются алгебраическими с однопорожденными компактными элементами.
\end{abstract}

Методы общей теории решеток широко используются в теории групп. Большое количество работ посвящено изучению тех или иных свойств решеток формаций и классов Фиттинга различных типов. Одним из таких свойств является алгебраичность решетки. В работах [1-3] показано, что решетка всех формаций, решетка всех $n$-кратно локальных формаций, решетка всех разрешимых тотально локальных формаций и решетка всех разрешимых тотально локальных классов Фиттинга являются алгебраическими, причем здесь компактные элементы - это соответствующие однопорожденные классы. Напомним, что полная решетка называется алгебраической, если любой ее элемент является решеточным объединением компактных элементов. Элемент $c$ полной решетки $L$ называется компактным, если для любого подмножества $X \subseteq L$ из неравенства $c \leqslant \sup _{L} X$ вытекает существование такого конечного подмножества $X_{0} \subseteq X$, что $c \leqslant \sup X_{0}$.

В данной работе установлена алгебраичность решетки всех классов Фиттинга, решетки всех $n$-кратно $\Omega$-расслоенных классов Фиттинга с направлением $\varphi, \psi_{0} \leqslant \varphi$, и решетки всех тотально канонических классов Фиттинга.

Рассматриваются только конечные группы. Необходимые определения и обозначения можно найти в $[1,4-6]$. В частности, $\Omega$ - непустой подкласс класса всех конечных простых групп $\mathfrak{T}, \Omega^{\prime}=\mathfrak{T} \backslash \Omega$. Все функции принимают одинаковые значения на изоморфных группах из их области определения. Функция

$$
f: \Omega \cup\left\{\Omega^{\prime}\right\} \rightarrow\{\text { классы Фиттинга групп }\}
$$

называется $\Omega R$-функцией, функция

$$
\varphi: \mathfrak{I} \rightarrow\{\text { непустые формации Фиттинга }
$$

называется $F R$-функцией. Класс Фиттинга

$$
\mathfrak{F}=\Omega R(f, \varphi)=\left(G: O^{\Omega}(G) \in f\left(\Omega^{\prime}\right) \text { и } G^{\varphi(A)} \in f(A) \text { для всех } A \in \Omega \cap K(G)\right)
$$

называется $\Omega$-расслоенным с $\Omega$-спутником $f$ и направлением $\varphi$. При рассмотрении различных направлений получаются различные классы Фиттинга. В частности, класс Фиттинга $\mathfrak{F}=\Omega R(f, \varphi)$ называется $\Omega$-свободным и обозначается $\mathfrak{F}=\Omega F r R(f)$, если 
$\varphi(A)=\psi_{0}(A)=G_{A^{\prime}}$ для любой $A \in \mathfrak{I}, \Omega$-каноническим и обозначается $\mathfrak{F}=\Omega K R(f)$, если $\varphi(A)=\psi_{2}^{\prime}(A)=\Im_{A} \mathbb{G}_{A^{\prime}}$ для любой $A \in \mathfrak{I}, \Omega$-биканоническим и обозначается $\mathfrak{F}=\Omega B R(f)$, если $\varphi(A)=\psi_{2}(A)=G_{A^{\prime}}$ для любой неабелевой $A \in \mathfrak{I}$ и $\varphi(A)=\psi_{2}(A)=\mathbb{G}_{A} \mathbb{G}_{A^{\prime}}$ для любой абелевой $A \in \mathfrak{I}$.

При $n \geqslant 1$ класс Фиттинга $\mathfrak{F}$ называется $n$-кратно $\Omega$-расслоенным с направлением $\varphi$, если $\mathfrak{F}$ имеет хотя бы один $\Omega$-спутник, все непустые значения которого являются $(n-1)$-кратно $\Omega$-расслоенными классами Фиттинга с тем же направлением $\varphi, 0$-кратно $\Omega$-расслоенным с направлением $\varphi$ считается всякий класс Фиттинга, класс Фиттинга $\mathfrak{F}$ называется тотально $\Omega$-расслоенным с направлением $\varphi$, если он $n$-кратно $\Omega$-расслоенный с направлением $\varphi$ для всех натуральных $n$.

Перейдем к изложению полученных результатов.

Через $\Omega R_{\varphi}^{n}\left(\Omega R_{\varphi}^{\infty}\right)$ обозначим множество всех $n$-кратно (тотально) $\Omega$-расслоенных классов Фиттинга с направлением $\varphi$.

Лемма 1. $\Omega R_{\varphi}^{n}$ - полная решетка классов Фиттинга.

Доказательство. Применим индукцию по $n$. При $n=0$ утверждение леммы непосредственно следует из определения класса Фиттинга.

Пусть $n=1$. По лемме 12 из [4] пересечение любой совокупности $\Omega R$-классов Фиттинга $\mathfrak{F}_{i}, i \in I$, является $\Omega R$-классом Фиттинга, причем если $\mathfrak{F}_{i}=\Omega R\left(f_{i}, \varphi\right)$, $i \in I$, то $\mathfrak{F}=\bigcap_{i \in I} \mathfrak{F}_{i}=\Omega R(f, \varphi)$, где $f=\bigcap_{i \in I} f_{i}$. Для $n>1$ предположим, что пересечение любой совокупности $\Omega R_{\varphi}^{n-1}$-классов Фиттинга есть $\Omega R_{\varphi}^{n-1}$-класс Фиттинга и $\mathfrak{F}_{i}=\Omega R\left(f_{i}, \varphi\right) \in \Omega R_{\varphi}^{n}, i \in I$, причем $f_{i}(A) \in \Omega R_{\varphi}^{n-1}$ для любой $A \in \Omega \cup\left\{\Omega^{\prime}\right\}$. Тогда по предположению индукции $f(A)=\bigcap_{i \in I} f_{i}(A) \in \Omega R_{\varphi}^{n-1}$ и по определению $\mathfrak{F}=\Omega R(f, \varphi) \in \Omega R_{\varphi}^{n}$. Таким образом, пересечение любой совокупности $\Omega R_{\varphi}^{n}$-классов Фиттинга есть $\Omega R_{\varphi}^{n}$-класс Фиттинга для любого натурального $n$.

По лемме 10 из [4] $\varnothing=\Omega R(h, \varphi)$, где $h(A)=\varnothing$ для всех $A \in \Omega \cup\left\{\Omega^{\prime}\right\}$ и $\varphi-$ произвольное направление. Тогда по индукции, учитывая строение спутника $h$, нетрудно показать, что $\varnothing \in \Omega R_{\varphi}^{n}$.

Для любого $\Omega \subseteq G$ справедливо равенство $\mathbb{G}=\Omega R(m, \varphi)$, где $m(A)=G$ для всех $A \in \Omega \cup\left\{\Omega^{\prime}\right\}, \varphi$ - произвольное направление. Действительно, для любой группы $G \in G$ имеем $G^{\varphi(A)} \triangleleft G \in G=m(A)$ для всех $A \in \Omega \cap K(G), O^{\Omega}(G) \triangleleft G \in G=m\left(\Omega^{\prime}\right)$. Следовательно, $G \in \Omega R(m, \varphi)$ и $G \subseteq \Omega R(m, \varphi)$. Обратно, так как рассматриваются только классы Фиттинга конечных групп, справедливо включение $\Omega R(m, \varphi) \subseteq G$. Таким образом, $G=\Omega R(m, \varphi)$. Опять по индукции, учитывая строение спутника $m$, нетрудно показать, что $\& \in \Omega R_{\varphi}^{n}$. Следовательно, $\Omega R_{\varphi}^{n}-$ полная решетка классов Фиттинга для любого $n \in \mathbf{N} \cup\{0\}$. Лемма доказана.

Следствие 1. $\Omega R_{\varphi}^{\infty}-$ полная решетка классов Фиттинга.

Лемма 2. Пусть $\mathfrak{X}$ - непустой класс групn. Тогда fit $\mathfrak{X}$ coстоит из групп, получаемых в результате применения конечного числа операчий $S_{n} u R \kappa$ группам из $\mathfrak{X}$.

Доказательство. Пусть

$$
\mathfrak{F}=\left\{G \in k_{1} \ldots k_{m} \mathfrak{X} \mid k_{i} \in\left\{S_{n}, R\right\}, i=1, \ldots, m, m \in \mathbf{N}\right\} .
$$

Покажем. что $\mathfrak{F}$ является классом Фиттинга. Пусть $N \triangleleft H \in \mathfrak{F}$. Тогда $N \triangleleft H \in$ $k_{1} \ldots k_{t} \mathfrak{X}$ и, значит, $N \in S_{n}(H) \subseteq S_{n} k_{1} \ldots k_{t} \mathfrak{X}$. Следовательно, $N \in S_{n} k_{1} \ldots k_{t} \mathfrak{X}$ и $N \in \mathfrak{F}$. Допустим, что $B=N_{1} N_{2}$, где $N_{i} \in \mathfrak{F}, N_{i} \triangleleft B, i=1,2$. Тогда $N_{1} \in k_{i_{1}} \ldots k_{i_{r}} \mathfrak{X}$, 
$N_{2} \in k_{j_{1}} \ldots k_{j_{s}} \mathfrak{X}$. Ввиду того, что $S_{n}$ и $R$ являются расширяющимися и монотонными, получаем включения $\mathfrak{X} \subseteq k_{j_{1}} \ldots k_{j_{s}} \mathfrak{X}, k_{i_{1}} \ldots k_{i_{r}} \mathfrak{X} \subseteq k_{i_{1}} \ldots k_{i_{r}} k_{j_{1}} \ldots k_{j_{s}} \mathfrak{X}, k_{j_{1}} \ldots k_{j_{s}} \mathfrak{X} \subseteq$ $k_{i_{1}} \ldots k_{i_{r}} k_{j_{1}} \ldots k_{j_{s}} \mathfrak{X}$. Таким образом, $N_{i} \in k_{i_{1}} \ldots k_{i_{r}} k_{j_{1}} \ldots k_{j_{s}} \mathfrak{X}, i=1,2$, а значит, $B=$ $N_{1} N_{2} \in R k_{i_{1}} \ldots k_{i_{r}} k_{j_{1}} \ldots k_{j_{s}} \mathfrak{X}$ и $B \in \mathfrak{F}$. Следовательно, $\mathfrak{F}$ является классом Фиттинга.

Так как $\mathfrak{X} \subseteq \mathfrak{F}$, значит, и fit $\mathfrak{X} \subseteq \mathfrak{F}$. По определению класс Фиттинга $k_{i}$-замкнут, поэтому $k_{i}$ fit $\mathfrak{X}=$ fit $\mathfrak{X}$. Так как $\mathfrak{X} \subseteq$ fit $\mathfrak{X}$ и $k_{i}$ монотонна, справедливы соотношения $k_{m} \mathfrak{X} \subseteq k_{m}$ fit $\mathfrak{X}=$ fit $\mathfrak{X}$. Продолжая этот процесс, получим, что $k_{1} \ldots k_{m} \mathfrak{X} \subseteq k_{1}$ fit $\mathfrak{X}=$ fit $\mathfrak{X}$ для любого $m \in \mathbf{N}$. Следовательно, $\mathfrak{F} \subseteq$ fit $\mathfrak{X}$, и значит, fit $\mathfrak{X}=\mathfrak{F}$. Лемма доказана.

\section{Теорема 1. Решетка всех классов Фиттинга является алгебраической.}

Доказательство. Любой класс Фиттинга является решеточным объединением всех своих однопорожденных подклассов Фиттинга. Покажем, что каждый однопорожденный класс Фиттинга $\mathfrak{F}=$ fit $G$ является компактным элементом в решетке всех классов Фиттинга.

Пусть

$$
\mathfrak{F}=\text { fit } G \subseteq \mathfrak{M}=\vee\left(\mathfrak{F}_{i} \mid i \in I\right)=\text { fit }\left(\bigcup_{i \in I} \mathfrak{F}_{i}\right)
$$

Тогда $G \in$ fit $\left(\bigcup_{i \in I} \mathfrak{F}_{i}\right)$. По лемме $2 G \in k_{1} \ldots k_{m}\left(\bigcup_{i \in I} \mathfrak{F}_{i}\right)$, где $k_{i} \in\left\{S_{n}, R\right\}$, $i=1, \ldots, m$. Индукцией по $m$ покажем, что сушествует конечное множество $J \subset I$ такое, что $G \in k_{1} \ldots k_{m}\left(\bigcup_{j \in J} \mathfrak{F}_{j}\right)$

Пусть $m=1$. Если $G \in S_{n}\left(\bigcup_{i \in I} \mathfrak{F}_{i}\right)$, то найдется $T \in \bigcup_{i \in I} \mathfrak{F}_{i}$ такая, что $G \triangleleft T$. Так как $T \in \bigcup_{i \in I} \mathfrak{F}_{i}$, существует хотя бы один $\mathfrak{F}_{j}, j \in I$, такой, что $T \in \mathfrak{F}_{j}$, и значит, $G \in S_{n}\left(\mathfrak{F}_{j}\right)$. Если $G \in R\left(\bigcup_{i \in I} \mathfrak{F}_{i}\right)$, то $G=T_{1} \ldots T_{t}$, причем $t$ - конечное число, $T_{r} \triangleleft G, T_{r} \in \bigcup_{i \in I} \mathfrak{F}_{i}, r=1, \ldots, t$. Следовательно, найдутся $\mathfrak{F}_{1}, \ldots, \mathfrak{F}_{t}$ такие, что $T_{1} \in \mathfrak{F}_{1}, \ldots, T_{t} \in \mathfrak{F}_{t}$. Значит, $T_{r} \in \mathfrak{F}_{1} \cup \ldots \cup \mathfrak{F}_{t}$ и $G \in R\left(\mathfrak{F}_{1} \cup \ldots \cup \mathfrak{F}_{t}\right)$.

Пусть утверждение верно для $m>1$. Докажем справедливость утверждения для $m+1$. Тогда $G \in k_{1} k_{2} \ldots k_{m+1}\left(\bigcup_{i \in I} \mathfrak{F}_{i}\right)=k_{1}(\mathfrak{S})$, где $\mathfrak{S}=k_{2} \ldots k_{m+1}\left(\bigcup_{i \in I} \mathfrak{F}_{i}\right)$. Если $G \in S_{n}(\mathfrak{S})$, то $G \triangleleft T \in \mathfrak{S}$. Тогда по индукщии существуют $1, \ldots, p \in I$ такие, что $T \in k_{2} \ldots k_{m+1}\left(\mathfrak{F}_{1} \cup \ldots \cup \mathfrak{F}_{p}\right)$. Следовательно, $G \in S_{n} k_{2} \ldots k_{m+1}\left(\mathfrak{F}_{1} \cup \ldots \cup \mathfrak{F}_{p}\right)$. Если $G \in R(\mathfrak{S})$, то $G=T_{1} \ldots T_{t}, t-$ конечное число, $T_{r} \triangleleft G, T_{r} \in \mathfrak{H}, r=1, \ldots, t$. По индукции существуют $r_{1}, \ldots, r_{k} \in I$ такие, что $T_{r} \in k_{2} \ldots, k_{m+1}\left(\mathfrak{F}_{r_{1}} \cup \ldots \cup \mathfrak{F}_{r_{k}}\right)$. Объ эдиняя найденные классы Фиттинга для каждого $T_{r}$, образуем класс $k_{2} \ldots k_{m+1}\left(\mathfrak{F}_{1} \cup \ldots \cup \mathfrak{F}_{p}\right)$, причем очевидно, что $k_{2} \ldots k_{m+1}\left(\mathfrak{F}_{r_{1}} \cup \ldots \cup \mathfrak{F}_{r_{k}}\right) \subseteq k_{2} \ldots k_{m+1}\left(\mathfrak{F}_{1} \cup \ldots \cup \mathfrak{F}_{p}\right)$ для всех групп $T_{r}$. Таким образом, $T_{r} \in k_{2} \ldots k_{m+1}\left(\mathfrak{F}_{1} \cup \ldots \cup \mathfrak{F}_{p}\right), r=1, \ldots, t$, и $G \in R k_{2} \ldots k_{m+1}\left(\mathfrak{F}_{1} \cup \ldots \cup \mathfrak{F}_{p}\right)$. Утверждение индукции доказано.

Поскольку $G \in k_{1} \ldots k_{m}\left(\bigcup_{j \in J} \mathfrak{F}_{j}\right) \in$ fit $\left(\bigcup_{j \in J} \mathfrak{F}_{j}\right), J-$ конечное множество из $I$, справедливо включение fit $G \subseteq$ fit $\left(\bigcup_{j \in J} \mathfrak{F}_{j}\right)$, и значит, $\mathfrak{F}$-компактный элемент решетки всех классов Фиттинга. Теорема доказана.

Пусть $\theta$ - полная решетка классов Фиттинга и $\mathfrak{X}$ - произвольная непустая совокупность групп. Пересечение всех $\theta$-классов Фиттинга, содержащих $\mathfrak{X}$, обозначим через $\theta$ fit $\mathfrak{X}$. Мы будем называть $\Omega$-радикальную функцию $\theta$-значной, или, коротко, $\Omega R \theta$-функцией, если все ее значения принадлежат $\theta$. Через $\Omega R_{\varphi} \theta$ обозначим множество всех $\Omega$-расслоенных классов Фиттинга с направлением $\varphi$, обладающих хотя бы одним $\Omega \theta$-спутником. Пересечение всех $\Omega R_{\varphi} \theta$-классов Фиттинга, содержащих $\mathfrak{X}$, обозначим через $\Omega R \theta(\mathfrak{X}, \varphi)$. 
Лемма 3. Пусть $\mathfrak{X}$ - непустой класс групп, $\theta$ - полная решетка классов Фиттинга. Тогда $\Omega$-расслоенный класс Фиттинга $\mathfrak{F}=\Omega R \theta(\mathfrak{X}, \varphi)$ с направлением $\varphi$, где $\psi_{0} \leqslant \varphi$, обладает единственным минимальным $\Omega \theta$-спутником $f$ таким, что

$$
\begin{aligned}
f\left(\Omega^{\prime}\right) & =\theta \operatorname{fit}\left(O^{\Omega}(G) \mid G \in \mathfrak{X}\right), & & \\
f(A) & =\theta \operatorname{fit}\left(G^{\varphi(A)} \mid G \in \mathfrak{X}\right), & & A \in \Omega \cap K(\mathfrak{X}), \\
f(A) & =\varnothing, & & A \in \Omega \backslash K(\mathfrak{X}) .
\end{aligned}
$$

Доказательство. Пусть $\mathfrak{X}$ - непустой класс групп, $\varphi$ - такая $F R$-функщия, что $\psi_{0} \leqslant \varphi$. Так как $\&-\Omega$-расслоенный класс Фиттинга с направлением $\varphi$, причем все значения его $\Omega$-спутника равны $\& \in \theta$, справедливо включение $\& \in \Omega R_{\varphi} \theta$. Кроме того, $\mathfrak{X} \subseteq G$, и значит, $\mathfrak{F}=\Omega R \theta(\mathfrak{X}, \varphi)$ сушествует и множество $L$ всех $\Omega \theta$-спутников $\mathfrak{F}$ непусто. Обозначим через $f_{1}$ пересечение всех элементов из $L$. Тогда по лемме 12 из [4] $\mathfrak{F}=\Omega R\left(f_{1}, \varphi\right)$. Так как $f_{1}(A)=\bigcap_{i \in I} f_{i}(A) \in \theta$, справедливо включение $f_{1} \in L$. Так как $f_{1} \leqslant f_{i}$ для любого $f_{i} \in L$, то $f_{1}$ - единственный минимальный $\Omega \theta$-спутник класса Фиттинга $\mathfrak{F}$.

Пусть $f-\Omega R$-функция, описанная в заключении леммы. Покажем, что $f=f_{1}$. Пусть $M \in \mathfrak{X}$. Тогда $O^{\Omega}(M) \in f\left(\Omega^{\prime}\right)$ и из $K(M) \subseteq K(\mathfrak{X})$ следует, что $M^{\varphi(A)} \in f(A)$ для всех $A \in \Omega \cap K(M)$. Значит, $M \in \Omega R(f, \varphi)$ и $\mathfrak{X} \subseteq \Omega R(f, \varphi)$. По построению $f-$ $\Omega \theta$-спутник, поэтому $\mathfrak{F}=\Omega R \theta(\mathfrak{X}, \varphi) \subseteq \Omega R(f, \varphi)$.

Аналогично теореме 10 из [4], учитывая, что $f_{1}-\Omega \theta$-спутник, получаем, что

$$
\begin{aligned}
f\left(\Omega^{\prime}\right) & =\theta \operatorname{fit}\left(O^{\Omega}(G) \mid G \in \mathfrak{X}\right) \subseteq f_{1}\left(\Omega^{\prime}\right), & & \\
f(A) & =\theta \operatorname{fit}\left(G^{\varphi(A)} \mid G \in \mathfrak{X}\right) \subseteq f_{1}(A), & & A \in \Omega \cap K(\mathfrak{X}), \\
f(A) & =\varnothing \subseteq f_{1}(A), & & A \in \Omega \backslash K(\mathfrak{X}) .
\end{aligned}
$$

Следовательно, $f \leqslant f_{1}$ и $\Omega R(f, \varphi) \subseteq \Omega R\left(f_{1}, \varphi\right)$. Тем самым установлено, что $\mathfrak{F}=\Omega R(f, \varphi)$, и значит, $f \in L$. Поскольку $f_{1}$ - единственный минимальный $\Omega \theta$-спутник класса Фиттинга $\mathfrak{F}$, из $f \leqslant f_{1}$ следует, что $f=f_{1}$. Лемма доказана.

Следствие 2. Пусть $\mathfrak{F}=K^{\infty} R\left(\mathfrak{X )}\right.$ и $f-$ минимальный $K^{\infty}$-значный спутник класса Фиттинга $\mathfrak{F}$. Тогда $f(A)=K^{\infty} R\left(O^{A, A^{\prime}}(G) \mid G \in \mathfrak{X}\right)$ для всех $A \in K(\mathfrak{X})$ u $f(A)=\varnothing$ для всех $A \in \mathfrak{I} \backslash K(\mathfrak{X})$.

Для произвольной совокупности $\theta$-классов Фиттинга $\left\{\mathfrak{F}_{i} \mid i \in I\right\}$ положим

$$
\vee^{*}\left(\mathfrak{F}_{i} \mid i \in I\right)=\theta \text { fit }\left(\bigcup_{i \in I} \mathfrak{F}_{i}\right)
$$

Пусть $\left\{f_{i} \mid i \in I\right\}-$ система $\Omega R \theta$-функций. Тогда через $\vee^{\theta}\left(f_{i} \mid i \in I\right)$ обозначим такую $\Omega R \theta$-функцию, что $\vee^{\theta}\left(f_{i} \mid i \in I\right)(A)=\vee^{\theta}\left(f_{i}(A) \mid i \in I\right)$ для любой $A \in \Omega \cup\left\{\Omega^{\prime}\right\}$.

Лемма 4. Пусть $\theta$ - полная решетка и $f_{i}-$ минимальный $\Omega \theta$-спутник $\Omega$-расслоенного класса Фиттинга $\mathfrak{F}_{i}$ с направлением $\varphi$, где $\psi_{0} \leqslant \varphi, i \in I$. Тогда $\vee^{\theta}\left(f_{i} \mid i \in I\right)-$ минимальный $\Omega \theta$-спутник класса Фиттинга $\mathfrak{F}=\vee^{\Omega R_{\varphi} \theta}\left(\mathfrak{F}_{i} \mid i \in I\right)$.

Доказательство. Пусть $f=\vee^{\theta}\left(f_{i} \mid i \in I\right)$ и $m$ - минимальный $\Omega \theta$-спутник класса Фиттинга $\mathfrak{F}=\vee^{\Omega R_{\varphi} \theta}\left(\mathfrak{F}_{i} \mid i \in I\right)$. Покажем, что $f=m$. 
Пусть $A \in\left\{\Omega^{\prime}\right\}$. Тогда, применяя лемму 3 , получим, что

$$
\begin{aligned}
m\left(\Omega^{\prime}\right) & =\theta \text { fit }\left(O^{\Omega}(G) \mid G \in \bigcup_{i \in I} \mathfrak{F}_{i}\right)=\theta \text { fit }\left(\bigcup_{i \in I} \theta \operatorname{fit}\left(O^{\Omega}(G) \mid G \in \mathfrak{F}_{i}\right)\right) \\
& =\theta \text { fit }\left(\bigcup_{i \in I} f_{i}\left(\Omega^{\prime}\right)\right)=\vee^{\theta}\left(f_{i}\left(\Omega^{\prime}\right) \mid i \in I\right)=f\left(\Omega^{\prime}\right) .
\end{aligned}
$$

Пусть $A \in \Omega \backslash K(\mathfrak{F})$. Тогда $m(A)=\varnothing$. Так как $\bigcup_{i \in I} \mathfrak{F}_{i} \subseteq \mathfrak{F}$, то $A \notin K\left(\bigcup_{i \in I} \mathfrak{F}_{i}\right)$, и значит, $A \notin K\left(\mathfrak{F}_{i}\right)$ и $f_{i}(A)=\varnothing$ для любого $i \in I$, так что $f(A)=\vee^{\theta}\left(f_{i}(A) \mid i \in I\right)=\varnothing$.

Если $A \in(\Omega \cap K(\mathfrak{F})) \backslash K\left(\cup_{i \in I} \mathfrak{F}_{i}\right)$, то, как и выше, $f(A)=m(A)=\varnothing$.

Пусть $A \in \Omega \cap K\left(\cup_{i \in I} \mathfrak{F}_{i}\right)$. Тогда существует $j \in I$ такое, что $A \in K\left(\mathfrak{F}_{j}\right)$, и значит, $f_{j}(A) \neq \varnothing$. Тогда, применяя лемму 3 , получим, что

$$
\begin{aligned}
m(A) & =\theta \text { fit }\left(G^{\varphi(A)} \mid G \in \bigcup_{i \in I} \mathfrak{F}_{i}\right)=\theta \text { fit }\left(\bigcup_{i \in I} \theta \operatorname{fit}\left(G^{\varphi(A)} \mid G \in \mathfrak{F}_{i}\right)\right) \\
& =\theta \text { fit }\left(\bigcup_{i \in I} f_{i}(A)\right)=\vee^{\theta}\left(f_{i}(A) \mid i \in I\right)=f(A) .
\end{aligned}
$$

Таким образом, $f(A)=m(A)$ для любой $A \in \Omega \cup\left\{\Omega^{\prime}\right\}$, и значит, $f=m$.

Лемма доказана.

В дальнейшем, если $\theta=\Omega R_{\varphi}^{n}\left(\Omega R_{\varphi}^{\infty}\right)$, то вместо символов $\vee^{\theta}\left(f_{i} \mid i \in I\right)$ и $\vee^{\theta}\left(\mathfrak{F}_{i} \mid i \in I\right)$ будем использовать символы $\vee^{n}\left(f_{i} \mid i \in I\right)\left(\vee^{\infty}\left(f_{i} \mid i \in I\right)\right)$ и $\vee^{n}\left(\mathfrak{F}_{i} \mid i \in I\right)\left(\vee^{\infty}\left(\mathfrak{F}_{i} \mid i \in I\right)\right)$. Те же символы будем использовать, если из контекста очевидно, о каком $\Omega$-расслоенном классе Фиттинга идет речь.

Теорема 2. Решетка $\Omega R_{\varphi}^{n}$, где $\psi_{0} \leqslant \varphi$, является алгебраической.

Доказательство. Так как любой $n$-кратно $\Omega$-расслоенный класс Фиттинга с направлением $\varphi, \psi_{0} \leqslant \varphi$, является объединением (в решетке $\Omega R_{\varphi}^{n}$ ) своих однопорожденных $\Omega R_{\varphi}^{n}$-подклассов Фиттинга, для доказательства утверждения достаточно показать, что каждый однопорожденный $\Omega R_{\varphi}^{n}$-класс Фиттинга $\mathfrak{F}$ является компактным элементом решетки $\Omega R_{\varphi}^{n}$. Применим индукцию по $n$. Пусть

$$
\mathfrak{F}=\Omega R^{n}(G, \varphi) \subseteq \mathfrak{M}=\vee^{n}\left(\mathfrak{F}_{i} \mid i \in I\right)=\Omega R^{n}\left(\bigcup_{i \in I} \mathfrak{F}_{i}, \varphi\right)
$$

где $\mathfrak{F}_{i}-n$-кратно $\Omega$-расслоенный класс Фиттинга с тем же направлением $\varphi, i \in I$.

При $n=0$ утверждение верно в силу теоремы 1 .

Пусть $n>0$ и однопорожденные $\Omega R_{\varphi}^{n-1}$-классы Фиттинга являются компактными элементами в решетке $\Omega R_{\varphi}^{n-1}$. Пусть $f_{i}$ - минимальный $\Omega R_{\varphi}^{n-1}$-спутник класса Фиттинга $\mathfrak{F}_{i}, m$ - минимальный $\Omega R_{\varphi}^{n-1}$-спутник класса Фиттинга $\mathfrak{M}, f-$ минимальный $\Omega R_{\varphi}^{n-1}$ спутник класса Фиттинга $\mathfrak{F}$. Тогда по теореме 1 из [7]

$$
f\left(\Omega^{\prime}\right)=\Omega R^{n-1}\left(O^{\Omega}(G), \varphi\right), \quad f(A)=\Omega R^{n-1}\left(G^{\varphi(A)}, \varphi\right)
$$

для всех $A \in \Omega \cap K(G), f(A)=\varnothing$, если $A \in \Omega \backslash K(G)$. Так как $\mathfrak{F} \subseteq \mathfrak{M}$, ввиду следствия 1.1 из [7] $f \leqslant m$. Согласно лемме $4 m=\vee^{n-1}\left(f_{i} \mid i \in I\right)$, и значит, по индукции, найдутся 
такие индексы $i_{1}, \ldots, i_{t} \in I$, что $O^{\Omega}(G) \in f\left(\Omega^{\prime}\right) \subseteq f_{i_{1}}\left(\Omega^{\prime}\right) \vee^{n-1} \ldots \vee^{n-1} f_{i_{t}}\left(\Omega^{\prime}\right)$, и для каждой $A \in \Omega \cap K(G)$ найдутся такие индексы $j_{1}, \ldots, j_{r} \in I$, что $G^{\varphi(A)} \in f(A) \subseteq$ $f_{j_{1}}(A) \vee^{n-1} \ldots \vee^{n-1} f_{j_{r}}(A)$.

Возьмем объединение всех этих индексов и обозначим их $k_{1}, \ldots, k_{s}$. Тогда по лемме $4 f_{k_{1}} \vee^{n-1} \ldots \vee^{n-1} f_{k_{s}}-$ минимальный $\Omega R_{\varphi}^{n-1}$-значный спутник класса Фиттинга $\mathfrak{F}_{k_{1}} \vee^{n} \ldots \vee^{n} \mathfrak{F}_{k_{s}}$. Получаем, что $O^{\Omega}(G) \in f\left(\Omega^{\prime}\right) \subseteq f_{k_{1}}\left(\Omega^{\prime}\right) \vee^{n-1} \ldots \vee^{n-1} f_{k_{s}}\left(\Omega^{\prime}\right)$, $G^{\varphi(A)} \in f(A) \subseteq f_{k_{1}}(A) \vee^{n-1} \ldots \vee^{n-1} f_{k_{s}}(A)$ для любой $A \in \Omega \cap K(G)$, и значит, $G \in \mathfrak{F}_{k_{1}} \vee^{n} \ldots \vee^{n} \mathfrak{F}_{k_{s}}$. Таким образом, $\mathfrak{F} \subseteq \mathfrak{F}_{k_{1}} \vee^{n} \ldots \vee^{n} \mathfrak{F}_{k_{s}}$. Следовательно, решетка $\Omega R_{\varphi}^{n}$ является алгебраической.

Теорема доказана.

Следствие 3. Решетки $\Omega F r^{n}, \Omega B^{n}, \Omega K^{n}$ являются алгебраическими.

Следствие 4. Решетки $F r^{n} . B^{n}, K^{n}$ являются алгебраическими.

Теорема 3. Решетка $K^{\infty}$ является алгебраической.

Доказательство. Любой тотально канонический класс Фиттинга является решеточным объединением всех своих однопорожденных тотально канонических подклассов Фиттинга. Покажем, что каждый однопорожденный $K^{\infty}$-класс Фиттинга $\mathfrak{F}=K^{\infty} R(G)$ является компактным элементом в решетке $K^{\infty}$.

Пусть $\mathfrak{F} \subseteq \mathfrak{M}=\vee^{\infty}\left(\mathfrak{F}_{i} \mid i \in I\right)$, где $\mathfrak{F}_{i}=K^{\infty} R\left(f_{i}\right)$, причем в качестве $f_{i}$ возьмем минимальный $K^{\infty}$-значный спутник класса Фиттинга $\mathfrak{F}_{i}, i \in I$.

Доказательство проведем индукцией по длине $l$ композиционного ряда группы $G \in \mathfrak{F}$. Если $l(G)=1$, то $G \cong A$ - простая группа, а значит, $O^{A, A^{\prime}}(G)=1$. Так как $G \cong A \in \mathfrak{F} \subseteq$ $\mathfrak{M}=K^{\infty} R\left(\bigcup_{i \in I} \mathfrak{F}_{i}\right)$, ввиду следствия 2 получаем, что $A \in K\left(\bigcup_{i \in I} \mathfrak{F}_{i}\right)=\bigcup_{i \in I} K\left(\mathfrak{F}_{i}\right)$, то есть сушествует такое $j \in I$, что $A \in K\left(\mathfrak{F}_{j}\right)$, и значит, $f_{j}(A) \neq \varnothing$. Тогда справедливы включения $O^{A, A^{\prime}}(G) \in f_{j}(A)$ и $G \in K^{\infty} R\left(f_{j}\right)$. Следовательно, $\mathfrak{F} \subseteq \mathfrak{F}_{j}$.

Пусть $l(G) \geqslant 1$ и все тотально канонические классы Фиттинга вида $K^{\infty} R(D)$, где $l(D)<l(G)$, являются компактными элементами решетки $K^{\infty}$. Обозначим через $f$ минимальный $K^{\infty}$-значный спутник класса $\mathfrak{F}, m-$ минимальный $K^{\infty}$-значный спутник класса $\mathfrak{M}$. Тогда по следствию $2 f(A)=K^{\infty} R\left(O^{A, A^{\prime}}(G)\right)$ для всех $A \in K(G)$ и $f(A)=\varnothing$, если $A \notin K(G)$. Так как $\mathfrak{F} \subseteq \mathfrak{M}$, из следствия 2 получаем, что $f \leqslant m$. Согласно лемме 4 $m=\vee^{\infty}\left(f_{i} \mid i \in I\right)$.

Рассмотрим композиционный ряд группы $G G=G_{0} \subset G_{1} \subset \ldots \subset G_{n}=1$. Тогда $G / G_{1} \cong A-$ простая группа, и значит, $O^{A, A^{\prime}}(G) \subseteq G_{1}$ и $l\left(O^{A, A^{\prime}}(G)\right)<l(G)$. Следовательно, по индукции найдутся такие индексы $i_{1}, \ldots, i_{t} \in I$, что $O^{A, A^{\prime}}(G) \in f(A) \subseteq$ $f_{i_{1}}(A) \vee^{\infty} \ldots \vee^{\infty} f_{i_{t}}(A)$. Так как $G / G_{1} \cong A=B^{\prime}$ для любой $B \in K(G) \backslash(A)$, справедливы соотношения $O^{B, B^{\prime}}(G) \subseteq G_{1}$ и $l\left(O^{B, B^{\prime}}(G)\right)<l(G)$. Значит, для каждой $B \in K(G) \backslash(A)$ найдутся такие индексы $j_{1}, \ldots, j_{r} \in I$, что $O^{B, B^{\prime}}(G) \in f(B) \subseteq f_{j_{1}}(A) \vee^{\infty} \ldots \vee^{\infty} f_{j_{r}}(A)$.

Возьмем объединение всех этих индексов и обозначим их $k_{1}, \ldots, k_{s}$. Тогда по лемме $4 f_{k_{1}} \vee^{\infty} \ldots \vee^{\infty} f_{k_{s}}-$ минимальный $K^{\infty}$-значный спутник класса Фиттинга $\mathfrak{F}_{k_{1}} \vee^{\infty} \ldots \vee^{\infty} \mathfrak{F}_{k_{s}}$. Так как $O^{C, C^{\prime}}(G) \in f(C) \subseteq f_{k_{1}}(C) \vee^{\infty} \ldots \vee^{\infty} f_{k_{s}}(C)$ для любой $C \in K(G)$, справедливо включение $G \in \mathfrak{F}_{k_{1}} \vee^{\infty} \ldots \vee^{\infty} \mathfrak{F}_{k_{s}}$. Таким образом, $\mathfrak{F} \subseteq \mathfrak{F}_{k_{1}} \vee^{\infty} \ldots \vee^{\infty} \mathfrak{F}_{k_{s}}$. Значит, $\mathfrak{F}$ - компактный элемент решетки $K^{\infty}$.

Теорема доказана.

Теорема 4. Пусть $\mathfrak{F}$ - однопорожденный тотально канонический класс Фиттинга. Тогда решетка всех тотально канонических подклассов Фиттинга в классе Фиттинга $\mathfrak{F}$ конечна. 
Доказательство. Пусть $\mathfrak{F}=K^{\infty} R(G)$. Доказательство проведем индукцией по длине $l$ композиционного ряда группы $G$.

Если $l(G)=1$, то $G \cong A-$ простая группа, и значит, $\mathfrak{F}=K^{\infty} R(A)=\mathfrak{G}_{A}$. Тогда решетка всех тотально канонических подклассов Фиттинга в $\mathfrak{F}$ конечна.

Пусть теперь $l(G) \geqslant 1$ и для всех тотально канонических классов Фиттинга вида $K^{\infty} R(D)$, где $l(D)<l(G)$, решетка их всех тотально канонических подклассов Фиттинга конечна.

Пусть $\mathfrak{M}$ - произвольный тотально канонический подкласс Фиттинга из $\mathfrak{F}$, и пусть $m$ и $f$ - минимальные $K^{\infty}$-значные спутники классов $\mathfrak{M}$ и $\mathfrak{F}$ соответственно. Тогда по следствию $2 f(A)=K^{\infty} R\left(O^{A, A^{\prime}}(G)\right)$ для всех $A \in K(G)$ и $f(A)=\varnothing$, если $A \notin K(G)$. Кроме того из включения $\mathfrak{M} \subseteq \mathfrak{F}$, ввиду следствия 2 , получаем, что $m \leqslant f$. Так как $l\left(O^{A, A^{\prime}}(G)\right)<l(G)$, решетка всех тотально канонических подклассов в $f(A)$ конечна. Так как множество $K(G)$ конечно, класс $\mathfrak{F}$ имеет лишь конечное множество тотально канонических подклассов Фиттинга.

Теорема доказана.

\section{Список литературы}

1. Скиба А. Н., Алгебра формачий. Беларуская навука, Минск, 1997.

2. Скиба А. Н., О локальных формациях длины 5. В кн.: Арифметическое и подгрупповое строение конечных групn. Наука и техника, Минск, 1986, с. 135-149.

3. Воробьев Н. Н., Скиба А. Н., Дистрибутивиость решетки разрешимых тотально локальных классов Фиттиіга. Препринт №82, Гомельский Гос. Унив., Гомель, 1999.

4. Ведерников В. А., Сорокина М. М., $\Omega$-расслоенные формации и классы Фиттинга конечных групп. Дискретиая математика (2001) 13, №3, 125-144.

5. Ведерников В. А., Максимальные спутники $\Omega$-расслоенных формаций и классов Фиттинга. Труды Матем. ии-та им. В. А. Стеклова РАН (2001) 2, 217-233.

6. Doerk K., Hawkes T., Finite soluble groupes. Gruyter, Berlin, 1992.

7. Сорокина М. М., О минимальных спутниках кратно $\Omega$-расслоенных классов Фиттинга и формаций конечных групп. В сб.: Бряпскому государствепюому педагогическому уииверситету 70 лет. БГПУ, Брянск, 2000, с. 199-203. 